\title{
Intratumoural PD-L1 expression is associated with worse survival of patients with Epstein- Barr virus-associated gastric cancer
}

An Na Seo ${ }^{1,6}$, Byung Woog Kang ${ }^{2,6}$, Oh Kyoung Kwon ${ }^{3}$, Ki Bum Park ${ }^{3}$, Seung Soo Lee ${ }^{3}$, Ho Young Chung ${ }^{3}$, Wansik $\mathrm{Yu}^{3}$, Han Ik Bae ${ }^{1}$, Seong Woo Jeon ${ }^{4}$, Hyojeung Kang ${ }^{5}$ and Jong Gwang Kim ${ }^{\star 2}$

${ }^{1}$ Department of Pathology, Kyungpook National University Medical Center, Kyungpook National University School of Medicine, 807 Hogukno, Buk-Gu, Daegu 41404, South Korea; ${ }^{2}$ Department of Oncology/Hematology, Kyungpook National University Hospital, Kyungpook National University School of Medicine, Kyungpook National University Cancer Research Institute, Kyungpook National University, 807 Hogukno, Buk-Gu, Daegu 41404, South Korea; ${ }^{3}$ Department of Surgery, Gastric Cancer Center, Kyungpook National University Medical Center, Kyungpook National University School of Medicine, 807 Hogukno, BukGu, Daegu 41404, South Korea; ${ }^{4}$ Department of Gastroeneterology, Kyungpook National University Medical Center, Kyungpook National University School of Medicine, 807 Hogukno, Buk-Gu, Daegu 41404, South Korea and ${ }^{5}$ College of Pharmacy, Institute of Microorganisms and Research Institute of Pharmaceutical Sciences, Kyunpook National University, 80 Daehakno, Buk-Gu, Daegu 41566, South Korea

Background: This study investigated the clinical relevance and prognostic impact of the overall expression of programmed cell death protein ligand-1 (PD-L1) and programmed cell death protein ligand-2 (PD-L2), in patients with Epstein-Barr virus-associated gastric cancer (EBVaGC).

Methods: After reviewing 1318 consecutive cases of surgically resected or endoscopic submucosal dissected gastric cancers, the expression status of PD-L1 and PD-L2 in 120 patients with EBVaGC identified by EBV-encoded RNA in situ hybridisation was retrospectively analysed using immunohistochemistry $(\mathrm{IHC})$. For each $\mathrm{IHC}$ marker, positivity was separately in intraepithelial tumour cells (iTu-) and immune cells in the tumour stroma area (str-).

Results: Among 116 eligible patients, 57 (49.1\%) and 66 patients (56.9\%) were determined as iTu-PD-L1-positive and str-PD-L1positive, respectively, whereas $23(21.6 \%)$ and 45 patients (38.8\%) were determined as iTu-PD-L2 positive and str-PD-L2 positive, respectively. Intraepithelial tumour cell PD-L1 positivity was found to be significantly associated with lymph node (LN) metastasis $(P=0.012)$ and a poor disease-free survival (DFS) $(P=0.032)$, yet not overall survival $(P=0.482)$. In a multivariate analysis, iTu-PD-L1 positivity was independently associated with a poor DFS $(P=0.006$, hazard ratio $=12.085)$. In contrast, str-PD-L2-positivity was related to a lower T category $(P=0.003)$, absence of $L N$ metastasis $(P=0.032)$ and perineural invasion $(P=0.028)$. Intraepithelial tumour cell and str-PD-L2 positivity showed a trend towards an improved DFS, although not significant $(P=0.060$ and $P=0.073$, respectively).

Conclusions: Intraepithelial tumour cells PD-L1 expression can be used to predict a poor outcome in patients with EBVaGC and can represent a rational approach for PD-1/PD-L pathway-targeted immunotherapy.

${ }^{\star}$ Correspondence: Professor JG Kim; E-mail: jkk21c@knu.ac.kr

${ }^{6}$ These authors contributed equally to this work.

Received 30 April 2017; revised 21 September 2017; accepted 22 September 2017; published online 26 October 2017

(C) 2017 Cancer Research UK. All rights reserved 0007-0920/17 
Immunotherapy has begun to revolutionise cancer treatment, by introducing therapies such as checkpoint inhibitors that target the host immune system instead of the tumour (Chen and Mellman, 2013). For an anticancer immune response, the programmed cell death protein-1 (PD-1) pathway is considered an important inhibitory mechanism regulating $\mathrm{T}$-cell exhaustion (Jin and Yoon, 2016). PD-1, which belongs to the CD28 family of proteins, is a receptor expressed on a number of immune cells, including $\mathrm{T}$ cells, B cells, monocytes, NK cells, and dendritic cells (Park et al, 2016). PD-1 has two ligands, the programmed cell death protein ligand-1 (PD-L1) and programmed cell death protein-ligand-2 (PD-L2). PD-L1 is expressed on T cells, B cells, dendritic cells, macrophages, mesenchymal stem cells, bone marrow-derived mast cells, and some non-haematopoietic cells, whereas PD-L2 is mainly expressed on antigen-presenting cells, including macrophages, dendritic cells, and non-haematopoietic tissues (Jin and Yoon, 2016). Several studies have already demonstrated that PD-L1 or PD-1 is highly expressed on tumour cells in gastric cancers (GCs) (Wu et al, 2006; Takaya et al, 2015; Zhang et al, 2015; Böger et al, 2016; Derks et al, 2016; Dong et al, 2016a; Eto et al, 2016; Kim et al, 2016; Takano et al, 2016; Cho et al, 2017). Interestingly, Wang et al (2016) suggested that GC patients with positive PD-L1 expression had a significantly shorter survival than PD-L1-negative patients, although the prognostic impact remained inconsistent.

The Cancer Genome Atlas data revealed that Epstein-Barr virus (EBV)-associated GC (EBVaGC) closely associated with a PIK3CA mutation, PD-L1/2 overexpression, EBV-CIMP, CDKN2A silencing, and immune cell signalling activation among GC subtypes. As previous studies have suggested that high $\mathrm{PD}-\mathrm{L} 1$ expression is related to a good responsiveness to anti-PD-1/PD-L1 therapy in human cancers, the EBVaGC subtype, in particular, may be a potential candidate for anti-PD-1/PD-L1 therapy among GC subtypes (Herbst et al, 2014; Taube et al, 2014). Furthermore, accumulating evidence suggests that tumour-infiltrating $\mathrm{T}$ cells may also be critical for the good responsiveness to anti-PD-1/PDL1 treatment (Teng et al, 2015). As EBVaGC is known to be associated with a prominent lymphoid infiltration of the stroma and high density of tumour-infiltrating lymphocytes (TILs), evaluating the PD-1 pathway affecting EBV infection may also be helpful in selecting appropriate targets for therapeutic interventions against EBVaGC. Moreover, this close relationship points to the possibility of PD-L1 and PD-L2 as prognostic markers in EBVaGC patients, suggesting a pivotal role of the immune mechanism in the EBVaGC subset. Several studies have reported PD-L1 expression in 34.4-92.5\% of EBVaGC patients (Derks et al, 2016; Dong et al, 2016a; Kawazoe et al, 2016; Ma et al, 2016). Notably, Saito et al (2016) recently reported that PD-L1 expression in tumour cells was associated with poor overall survival (OS) and disease-specific survival in 96 patients with EBVaGC. However, unfortunately, detailed clinicopathologic characteristics of EBVaGC showing PD-L1 have not been fully elucidated and no published study has yet investigated the clinical significance and prognostic impact of PD-L2 expression in EBVaGC.

Accordingly, this study used a relatively large series of EBVaGC to determine the clinical and prognostic significance of PD-L1 and PD-L2 in both tumour cells and immune cells, and examine the association with the density of TILs.

\section{MATERIALS AND METHODS}

Patients and samples. A total of 120 EBVaGC cases were used in this study, all of which were obtained from our previous studies (Kang et al, 2016). The inclusion criteria for patient selection and results have already been reported (Kang et al, 2016). Briefly, all the patients were histologically diagnosed with gastric adenocarcinoma and underwent surgical resection or endoscopic mucosal dissection (ESD) at Kyungpook National University Medical Center (KNUMC) between January 2011 and November 2014. Their status as EBV positive was determined by EBV-encoded RNA in situ hybridisation conducted at our molecular pathology laboratory. The baseline characteristics of the 120 patients are presented in Supplementary Table 1. The clinical and pathologic information was collected based on a review of the patients' medical records and pathologic reports, respectively. The patients were all staged according to the seventh edition of the American Joint Committee on Cancer staging Manual for Stomach cancer (Edge et al, 2010).

The Institutional Review Boards of Kyungpook National University Medical Center (IRB No.: KNUMC 2016-05-012) approved this study. The Reporting Recommendations for Tumour marker Prognostic Studies criteria were followed when reporting the results of this study (McShane et al, 2005).

Pathologic quantification of TILs. The evaluation of the percentage of intratumoural TILs (iTu-TILs) and stromal TILs (str-TILs) used a modified version of the TIL scoring recommendations of the International TILs Working Group 2014 on breast cancer (Dieci et al, 2014). The detailed quantification definition has been previously described (Kang et al, 2016). Specifically, the iTuTILs were defined as the percentage of mononuclear inflammatory cells within an intraepithelial tumour cell nest and in direct contact with tumour cells. Meanwhile, str-TILs were defined as the percentage of tumour stroma of invasive carcinoma to be occupied by mononuclear inflammatory cells. The percentage of TILs was evaluated in $10 \%$ increments; if the percentage of iTu-TILs and strTILs was $<10 \%, 1 \%$, or $5 \%$ criteria were used on full sections of haematoxylin and eosin-stained slides, and evaluated by two pathologists (ANS and HIB). The quantification of iTu-TILs and str-TILs was identified based on our previously published data (Kang et al, 2016).

Immunohistochemistry and interpretation. All the tissue slides were stained using an automatic immunohistochemistry (IHC) staining instrument (BenchMarkXT, Ventana Medical Systems, Tucson, AZ, USA) according to the manufacturer's instructions. The following primary antibodies were used: PD-L1 (E1L3N; 1 : 100; Cell Signaling Technology, Danvers, MA, USA) and PD-L2 (clone 176611; $1: 150$; R\&D Systems, Minneapolis, MN, USA). In brief, a representative formalin-fixed, paraffin-embedded tissue sample for each case was cut into $4 \mu \mathrm{m}$-thick sections, transferred to poly-L-lysine-coated adhesive slides, and dried in a microwave at $60{ }^{\circ} \mathrm{C}$ for $1 \mathrm{~h}$. Therefore, the sections were deparaffinised using an EZ Prep solution (Ventana Medical Systems). The cell-conditioning solution 1 (CC1) standard ( $\mathrm{pH} 8.4$ buffer containing Tris/ borate/EDTA) was then used for antigen retrieval, followed by blocking with inhibitor $\mathrm{D}\left(3 \% \mathrm{H}_{2} \mathrm{O}_{2}\right)$ at $37^{\circ} \mathrm{C}$ for $4 \mathrm{~min}$. Next, the slides were incubated with the primary antibodies at $37^{\circ} \mathrm{C}$ for $60 \mathrm{~min}$. The staining was optimised using a positive and negative control for each marker according to the recommendation of the supplier's data sheet. All the sections were visualised with an UltraView Universal DAB detection kit (Ventana Medical Systems).

The expression of PD-L1 and PD-L2 was evaluated by two experienced gastrointestinal pathologists (ANS and HIB) with no prior knowledge of the clinical data. The stained slides were semiquantitatively scored for the intensity and percentage of membranous and/or cytoplasmic staining of the tumour cells and tumour stoma area (immune cells), respectively. The interpretation of each marker was based on the following criteria: 0 , no expression or expression in $<1 \%$; $1+$, weak/faint expression in $\geqslant 1 \% ; 2+$, moderate expression in $\geqslant 1 \% ; 3+$, strong expression $\geqslant 1 \%$ of iTu- or immune cells in tumour str-. The cases were then classified 
into dichotomous covariates: negative $(0$ and $1+)$ and positive $(2+$ and $3+)$.

Statistical analyses. The descriptive statistics are reported as proportions and medians. The categorical variables were evaluated using a $\chi^{2}$-test, Fisher's exact test, or Pearson's correlation $(R)$ test, as appropriate. Disease-free survival was measured from the time of surgery or ESD to the time of tumour recurrence or death from any cause. Overall survival was calculated from the date of surgery or ESD to death from any cause. Data were censored if patients were free of recurrence or alive at the last follow-up. The patient subgroups were compared with respect to disease-free survival (DFS) and OS with the use of Kaplan-Meier curves, log-rank test, and multivariate survival analysis based on the Cox proportional hazard regression model. Hazard ratios (HRs) and 95\% confidence intervals (CIs) were estimated for each factor. All the tests were two-sided and statistical significance was set at $P<0.05$. All the statistical analyses were performed using SPSS for Windows (version 19.0, SPSS Inc., Chicago, IL, USA).

\section{RESULTS}

PD-L1 and PD-L2 expression. PD-L1 and PD-L2 IHC results were available for 116 of the 120 cases (4 cases were excluded due to loss of tissue or poor IHC staining). Immunohistochemical expression of PD-L1 and PD-L2 was observed in tumour cells (iTu-) and/or the immune cells in tumour stromal area (str-) in EBVaGC tissues (Figure 1). Intraepithelial tumour cell PD-L1 positivity was detected in 57 of the 116 EBVaGC cases $(49.1 \%)$ $(2+$ in 39 and $3+$ in 18 cases), whereas str-PD-L1 positivity was observed in 66 cases $(56.9 \%)$ ( $2+$ in 65 cases and $3+$ in 1 case). In addition, EBVaGC exhibiting both iTu-PD-L1 and str-PD-L1 was observed in 26 cases (22.4\%). Meanwhile, iTu-PD-L2 positivity was detected in 25 cases $(21.6 \%)$ ( $2+$ in all cases), whereas str-PDL2 positivity was observed in 45 cases $(38.8 \%)$ ( $2+$ in all cases). In addition, EBVaGC exhibiting both iTu-PD-L2 and str-PD-L2 was observed in 14 cases (12.1\%).
Association with clinicopathologic features and TILs. As described in Table 1, iTu-PD-L1 positivity was significantly associated with the presence of lymph node (LN) metastasis $(P=0.012)$, whereas str-PD-L1 positivity was related to categories associated with dense TILs including str-TIL positivity $(P=0.003)$, iTu-TIL positivity $(P=0.004)$, and gastric carcinoma with lymphoid stroma (GCLS) $(P=0.024)$. In contrast, str-PD-L2 positivity were correlated with a lower pT category $(P=0.003)$ and the absence of LN metastasis $(P=0.0032)$ and perineural invasion $(P=0.028)$, str-TIL positivity $(P<0.001)$, iTu-TIL positivity $(P<0.001)$, GCLS $(P<0.001)$, and a non-conventional adenocarcinoma subclassification $(P<0.001)$. However, iTu-PDL2 positivity showed no association with any clinicopathologic characteristics.

To determine the relationship between PD-L1 and PD-L2 expression in EBVaGC, we analysed the correlation between the levels of PD-L1 and PD-L2 expression (Table 2 and Supplementary Figure 1). The iTu expression of PD-L1 and PD-L2 was correlated with the str expression of PD-L1 and PD-L2 with each other, respectively (iTu-PD-L1 positivity and str-PD-L1: $R=-0.224$, $P=0.016 ; \quad$ iTu-PD-L1 positivity and str-PD-L1: $R=0.185$, $P=0.047)$.

Patient outcomes. At the time of the last follow-up (April 2016), the median follow-up duration for the survival analyses was 36.2 months (range 6.4-66.6). The probability of OS and DFS at 3 years was $92.2 \%$ and $88.5 \%$, respectively. During the analyses, $13(10.8 \%)$ patients experienced recurrence and $13(10.8 \%)$ died. In the univariate analysis, iTu-PD-L1 positivity was significantly associated with a worse DFS $(P=0.032)$ (Figure $2 \mathrm{~A})$, yet not with $\mathrm{OS}$ $(P=0.482)$. In the multivariate analysis using the Cox proportional hazard model adjusted for conventional clinical covariates, iTuPD-L1 positivity was an independent worse prognostic factor for DFS ( $\mathrm{HR}=12.085,95 \% \mathrm{CI}=2.013-72.559, P=0.006)$ (Table 3 ). Meanwhile, both iTu-PD-L2 positivity and str-PD-L2 positivity tended to show a favourable DFS (iTu-PD-L2: $P=0.060$; str-PDL2: $P=0.073$, yet without statistical significance in the univariate analysis (Figure $2 \mathrm{~B}$ and $\mathrm{C}$ ).
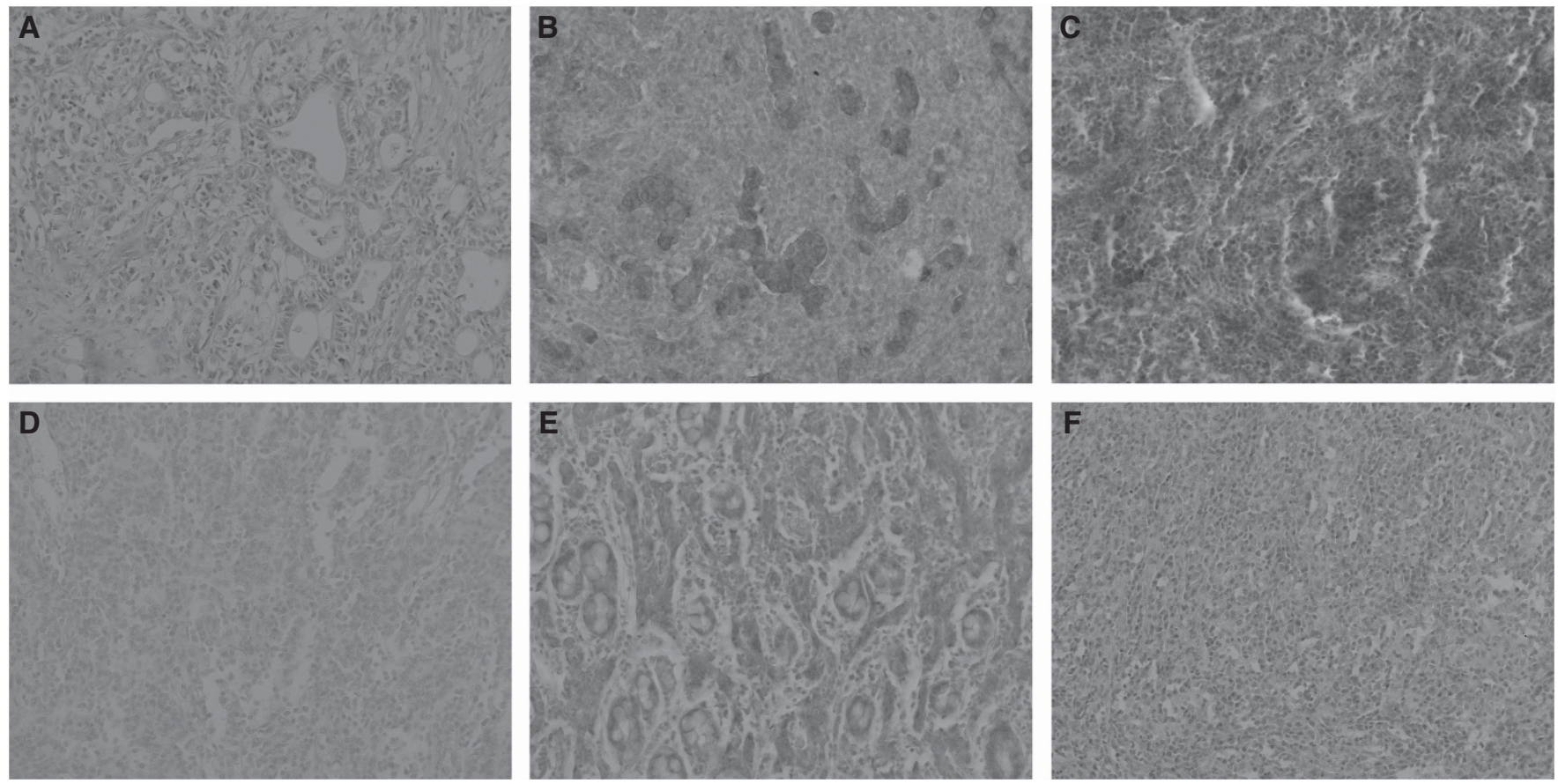

Figure 1. Representative features of immunohistochemical expression of PD-L1 and PD-L2 in EBV-associated gastric cancers. (A) Representative photographs of intraepithelial tumour cells (iTu-) or immune cells in tumour stroma area (str-)-PD-L1 negativity; (B) iTu-PD-L1 positivity; (C) str-PDL1 positivity. (D) Representative picture of iTu- or str-PD-L2 negativity; (E) iTu-PD-L2 positivity; (F) str-PD-L2 positivity. 
Table 1. The association between PD-L1/PD-L2 expression and clinicopathologic features

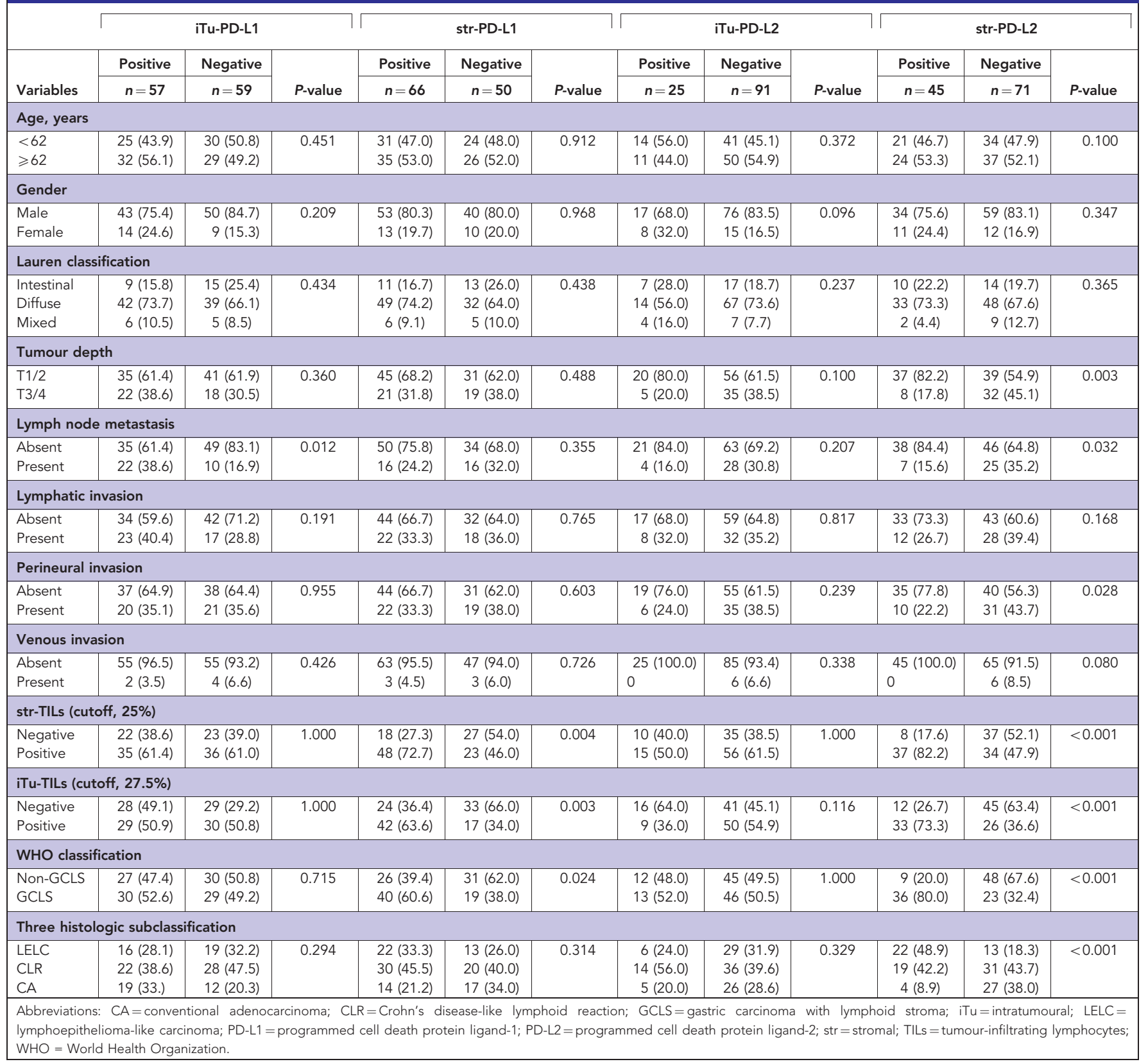

As str-TILs were also associated with DFS, the patients were further categorised into three groups (iTu-PD-Ll negativity and str-TIL positivity, and iTu-PD-L1 positivity and str-TIL negativity, and others). In univariate analysis, the patient groups with iTuPD-L1 positivity and str-TIL negativity showed a shorter DFS than the other groups $(P=0.006)$ (Figure 2D).

\section{DISCUSSION}

Accumulating data have revealed that PD-L1 expression can be a potential prognostic and therapeutic predictors for anti-PD-1/PDL1 immunotherapy, therefore, understand the implications of PDL1 expression in patients with EBVaGC is of critical importance (Jin and Yoon, 2016). However, as the detailed implications of PD-L1 and PD-L2 expression, two main ligands of PD-1, remain unclear in patients with EBVaGC, the present study investigated the clinicopathologic significance and prognostic value. As a result, the following key points were identified: iTu-PD-L1 positivity was significantly correlated with LN metastases and a poor prognosis, whereas str-PD-L2 positivity was inversely related to tumour invasion and LN metastasis. Notably, iTu-PD-L1 positivity was also identified as an independent prognostic factor of worse DFS. Furthermore, when combining iTu-PD-L1 and str-TILs, the patient with iTu-PD-L1 positivity and str-TIL negativity was associated with worse DFS when compared with the other patients with EBVaGC. Therefore, these findings support the concept that increased PD-L1 expression in tumours and intense lymphocytic infiltration around tumours can act as an immune modulator, promoting escape from immune surveillance in patients with EBVaGC.

The PD-1 pathway is considered a critical inhibitory mechanism regulating T-cell exhaustion (Jin and Yoon, 2016). In tumour beds, $\mathrm{T}$ cells must pass through numerous barriers with immune checkpoints, such as PD-1 and PD-L1/PD-L2, as intrinsic regulators (Palucka and Coussens, 2016). The PD-1 pathway is involved in suppressing autoimmunity during T-cell activation, 
allowing for immune tolerance of PD-L1- or PD-L2-expressed cells, with a blockade leading to T-cell rescue in subsequent response activation (Pardoll, 2012). Interestingly, PD-L1 mRNA and protein can be upregulated by cytokines produced by infiltrating immune cells (Dong et al, 2016b). Plus, oncogenic signalling pathways in tumour cells can activate PD-L1 expression (Dong et al, 2016b). Fang et al (2014) already reported that PD-L1 expression could be increased by exogenous and endogenous induction of Latent membrane protein 1 (LMP1)-induced PD-L1 and was suppressed by knocking down LMP1 in EBV-positive cell lines. Therefore, these mechanisms influence the regulation of virus immunity, as well as the cancer immune reaction in EBVaGC. To date, several studies have already shown that PD-L1 expression is

Table 2. The correlation between the levels of PD-L1 and PD-L2 expression

\begin{tabular}{|l|c|c|c|c|}
\hline & iTu-PD-L1 & Str-PD-L1 & iTu-PD-L2 & Str-PD-L2 \\
\cline { 2 - 5 } & $\boldsymbol{R}(\boldsymbol{P}$-value) & $\boldsymbol{R}(\boldsymbol{P}$-value $)$ & $\boldsymbol{R}(\boldsymbol{P}$-value $)$ & $\boldsymbol{R}(\boldsymbol{P}$-value) \\
\hline iTu-PD-L1 & - & $-0.224(0.016)$ & $0.114(0.224)$ & $-0.146(0.119)$ \\
\hline Str-PD-L1 & $-0.224(0.016)$ & - & $-0.094(0.315)$ & $0.086(0.361)$ \\
\hline iTu-PD-L2 & $0.114(0.224)$ & $-0.094(0.315)$ & - & $0.185(0.047)$ \\
\hline Str-PD-L2 & $-0.146(0.119)$ & $0.086(0.361)$ & $0.185(0.047)$ & - \\
\hline $\begin{array}{l}\text { Abbreviations: iTu = intratumoural; PD-L1 = programmed cell death protein ligand-1; PD- } \\
\text { L2= programmed cell death protein ligand-2; } R=\text { Pearson's correlation; str = stromal. }\end{array}$ \\
\hline
\end{tabular}
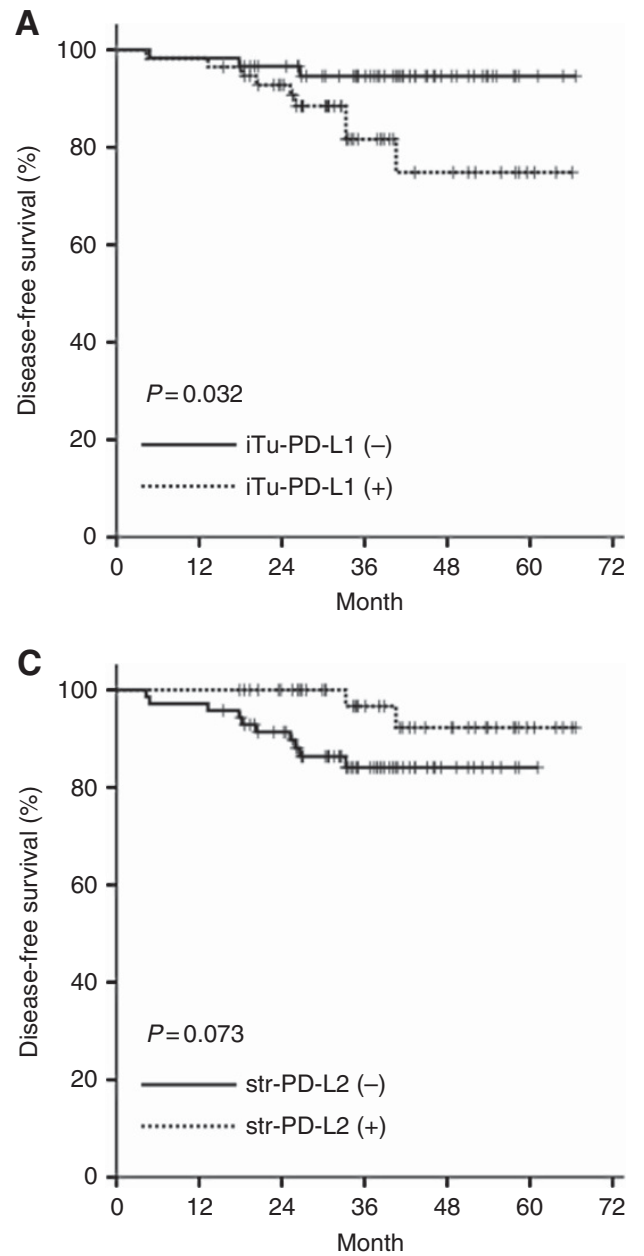

associated with a worse prognosis in GC. According to a recent meta-analysis by Zhang et al (2016) of 1901 GC patients based on 10 studies, PD-L1 expression was identified as a valuable predictor of poor OS with a final HR for OS of 1.64. Recently, low PD-L1 expression and high CD8 + TILs were associated with better OS and DFS in 392 patients with surgically resected GC. Although this study included only 25 patients with EBVaGC, group with increased PD-L1 expression and high CD8 + TILs was closely correlated with EBV infection (Koh et al, 2017). However, there is still insufficient evaluation of PD-L1 expression in patients with EBVaGC. Furthermore, to the best of our knowledge, there is no previously reported evaluation of PD-L2 in EBVaGC. So far, the association between PD-L1 expression and prognosis has been inconsistent across various studies (Table 4). These conflicting results could be due to different patient ethnicities and different antibody clones and interpretation. Indeed, previous studies have only focused on the frequency of PD-L1 expression and not the impact on the survival outcomes of EBVaGC. In consistent with the current study, Saito et al (2016) reported that the PD-L1positive EBVaGC patients exhibited a deeper invasion of the tumour and poorer prognosis than the PD-L1-negative patient. These authors also demonstrated that PD-L1-amplified cells corresponded to PD-L1-positive cells, showing high-intensity immunohistochemical cells. In contrast, another recent study found no association between PD-L1 expression and patient outcomes (Dong et al, 2016a). Consequently, the present findings need to be validated though further studies, in order to clarify the
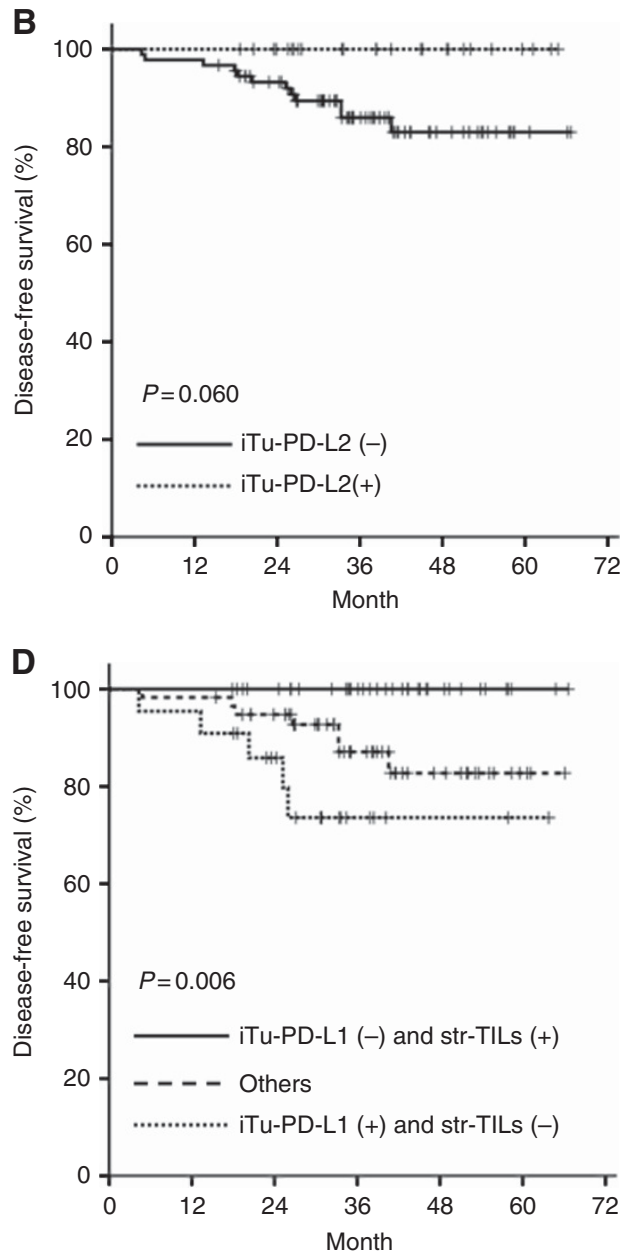

Figure 2. Survival analysis. (A) Kaplan-Meier survival curves for disease-free survival of patients stratified as iTu-PD-L1 positivity vs iTu-PD-L1 negativity, (B) iTu-PD-L2 positivity vs iTu-PD-L2 negativity, and (C) str-PD-L2 positivity vs str-PD-L2 negativity. (D) Kaplan-Meier survival curves for disease-free survival according to the three groups (iTu-PD-LI negativity and str-TIL positivity, and iTu-PD-L1 positivity and str-TIL negativity, and others). 
Table 3. Multivariate analysis for disease-free survival

\begin{tabular}{|c|c|c|c|c|}
\hline \multirow[b]{2}{*}{ Variables } & \multirow[b]{2}{*}{ Category } & \multicolumn{3}{|c|}{ Disease-free survival in all patients } \\
\hline & & $P$ & HR & $95 \% \mathrm{Cl}$ \\
\hline Gender & Female vs male & 0.250 & 3.879 & $0.385-39.115$ \\
\hline pTNM stage & I vs || and III & 0.014 & 13.863 & $1.688-113.847$ \\
\hline Venous invasion & Negative vs positive & 0.027 & 0.055 & $0.004-0.715$ \\
\hline Perineural invasion & Negative vs positive & 0.096 & 4.702 & $0.762-29.034$ \\
\hline Str-TILs & $\geqslant 25$ vs $<25$ & 0.647 & 1.982 & $0.106-36.975$ \\
\hline iTu-PD-L1 & Negative vs positive & 0.006 & 12.085 & $2.013-72.559$ \\
\hline
\end{tabular}

\section{Table 4. Frequency and prognostic value of PD-L1 expression in EBVaGC}

\begin{tabular}{|c|c|c|c|c|c|c|c|}
\hline References & Tumour type & Sample size & Country & $\begin{array}{c}\text { PD-L1 } \\
\text { expression }\end{array}$ & $\begin{array}{l}\text { Criteria } \\
\text { (cut-off) }\end{array}$ & Antibody & Prognostic role \\
\hline Saito et al (2016) & EBVaGC & 96 & Japan & $34.4 \%^{\mathrm{a}}$ & $>5 \%$ & $\begin{array}{c}\text { Clone E1L3N, Cell } \\
\text { Signaling }\end{array}$ & OS and DSS ${ }^{b}$ \\
\hline Derks et al (2016) & EBVaGC & 32 & USA & $50 \%^{a}$ & $>5 \%$ & Clone 405.9A11 & Not reported ${ }^{c}$ \\
\hline Dong et al (2016a) & EBVaGC & 59 & China & $92.5 \%^{a}$ & $>9.0^{d}$ & Ab58810, Abcam & No significant \\
\hline Kawazoe et al (2016) & EBVaGC & 25 & Japan & $52.0 \%^{a}$ & $\geqslant 1 \%$ & SP142, Ventana & Not reported \\
\hline Ma et al (2016) & $\mathrm{EBVaGC}$ & 7 & USA & $71.4 \%^{a}$ & $\geqslant 5 \%$ & SP263, Ventana & Not reported \\
\hline Current study & EBVaGC & 116 & South Korea & $47.5 \%^{a}$ & $\geqslant 1 \%$ & $\begin{array}{c}\text { Clone E1L3N, Cell } \\
\text { Signaling }\end{array}$ & DFS \\
\hline \multicolumn{8}{|c|}{ 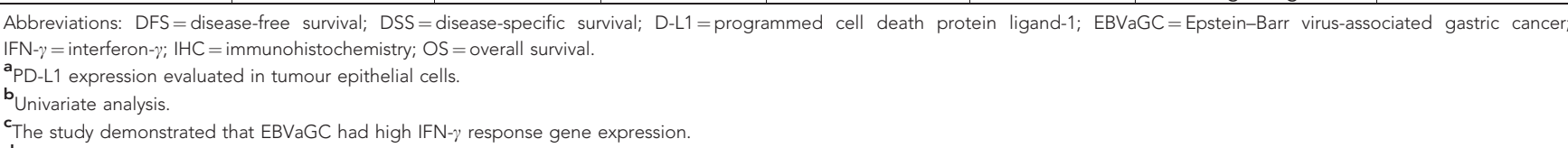 } \\
\hline
\end{tabular}

association between PD-L1 expression and the prognosis of EBVaGC.

Another interesting finding from the present study was that iTu-PD-L2- and str-PD-L2 positivity was tentatively associated with a favourable DFS and OS, although not significant in the EBVaGC patients. In contrast to PD-L1, PD-L2 expression is regulated by IL-4 and STAT6 on dendritic cells and macrophages, and PD-L2 have different functions under different Th1/Th2 inflammatory situations, even though PD-L1 and PD-L2 bind PD1 together (Loke and Allison, 2003). However, despite increasing evidence that PD-L2 has important roles in several aspects of cancer, it is still unclear whether PD-L2 itself alters the function of the PD-1 pathway in cancer (Zhang et al, 2006). Some studies have shown a stimulatory role for PD-L2, whereas others suggest that PD-L2 can inhibit T-cell activation (Latchman et al, 2001; Wang et al, 2003; Jin and Yoon, 2016). In the present study, str-PD-L2 expression was positively correlated with dense iTu- and/or strTILs, yet not iTu-PD-L2 expression. Ohigashi et al (2005) suggested that PD-L2 expression inversely correlated with tumour-infiltrating CD8 + T cells in 41 patients with oesophageal cancer. Thus, whether PD-L1 and PD-L2 are influenced by specific markers of TILs, such as CD4, CD8, and FOXP3, and interferon- $\gamma$ would be an interesting focus for further studies of the tumour immunity of EBVaGC. Meanwhile, for the correlation between PD-L1 and PD-L2 expression, PD-L1 was found to be expressed independently of PD-L2 expression. This finding supports previous report, where iTu-PD-L2 expression was induced in the absence of PD-L1 co-expression tumours, in which case PD-L2 expression was less likely to possess PD-L1 + immune cells (Derks et al, 2015). These observations can also help to understand the exact role of PD-L2 on the natural outcomes of EBVaGC.

TILs could be used to identify a subgroup of patients with excellent outcomes (Chang et al, 2014) and our previous study also showed an independent association between a high density of TILs and a favourable recurrence-free survival or DFS in 120 patients with EBVaGC (Kang et al, 2016). This finding supports that TILs exhibit a host cellular immune response against tumours, indicating that immunotherapy may have a potential role in patients with EBVaGC (Kang et al, 2015). Several studies have already demonstrated a correlation between TILs and PD-L1 expression in EBVaGC (Kawazoe et al, 2016; Ma et al, 2016; Saito et al, 2016). Interestingly, when combining the iTu-PD-L1 expression and TIL score, the patient group with iTu-PD-L1 negativity and str-TIL positivity was associated with a better DFS than the other EBVaGC patients. This result creates a very meaningful subgroup that can provide more information for tailored therapy for each group of patients. In particular, this finding can provide a novel strategy for subgroups with decreased immunoediting via the PD-1 pathway and a strengthened immune response by TILs, and vice versa, considering that the state of the 
immune equilibrium is a critical factor for therapeutic success (Chen and Mellman, 2013; Palucka and Coussens, 2016). In fact, alterations of TILs have already been implicated in immune homeostasis, as well as immune response in the $\mathrm{PD}-1$ pathway (Menon et al, 2016).

Although the present data identified a significant prognostic role of PD-L1 expression in operable EBVaGC, these results should be cautiously interpreted due to potential limitations. The current study is a retrospective evaluation, and the evaluation and interpretation of PD-L1 and PD-L2 within tumours and/or a tumour microenvironment have not yet been standardised in GCs (Manson et al, 2016). Moreover, a standardised cutoff value for PD-L1 - and PD-L2 positivity has not yet been clearly established (Festino et al, 2016), plus PD-L1 and PD-L2 expression cannot be used to predict treatment response to anti-PD-1/PD-L1 therapy in patients with EBVaGC. Nevertheless, the present study has several important differences with previous studies as follows: (1) a relatively large-scale cohort of EBVaGC patients with a Korean homogeneous ethnic identity (Jin and Yoon, 2016), (2) equivalent treatment application, (3) minimal loss to follow-up, (4) assessment of TILs, and (5) comprehensive interpretation of expression in cancer cells and immune cells as separate microenvironments of cancer cells.

\section{CONCLUSION}

In conclusion, str-PD-L1- and str-PD-L2 positivity was shown to be associated with dense iTu- and str-TILs in patients with EBVaGC. The current study also revealed significant prognostic impact of iTu-PD-L1 positivity by demonstrating an independent association with a poor DFS. Therefore, these findings support the concept that PD-L1 can be a prognostic indicator for predicting patient outcomes and a rationale for therapeutic targeting in EBVaGC.

\section{ACKNOWLEDGEMENTS}

The biospecimens for this study were provided by National Biobank of Korea-Kyungpook National University Hospital (KNUH), which is supported by the Ministry of Health, Welfare, and Affairs. All materials derived from the National Biobank of Korea-KNUH were obtained (with informed consent) under institutional review board (IRB)-approved protocols. This work was supported by the National Research Foundation of Korea (NRF) grant funded by the Korea government (2014R1A5A2009242) and Basic Science Research Program through the National Research Foundation of Korea (NRF) funded by the Ministry of Education (2017R1D1A3B03032320).

\section{CONFLICT OF INTEREST}

The authors declare no conflict of interest.

\section{REFERENCES}

Böger C, Behrens H-M, Mathiak M, Krüger S, Kalthoff H, Röcken C (2016) $\mathrm{PD}-\mathrm{L} 1$ is an independent prognostic predictor in gastric cancer of Western patients. Oncotarget 7(17): 24269-24283.

Chang W-J, Du Y, Zhao X, Ma L-Y, Cao G-W (2014) Inflammation-related factors predicting prognosis of gastric cancer. World J Gastroenterol 20(16): 4586-4596.

Chen DS, Mellman I (2013) Oncology meets immunology: the cancerimmunity cycle. Immunity 39(1): 1-10.
Cho J, Lee J, Bang H, Tae Kim S, Hoon Park S, Yeong An J, Gew Choi M, Ho Lee J, Sung Sohn T, Moon Bae J, Ki Kang W, Kim S, Kim K-M (2017) Programmed cell death-ligand 1 expression predicts survival in patients with gastric carcinoma with microsatellite instability. Oncotarget 8(8): 13320-13328.

Derks S, Liao X, Chiaravalli AM, Xu X, Camargo MC, Solcia E, Sessa F, Fleitas T, Freeman GJ, Rodig SJ, Rabkin CS, Bass AJ (2016) Abundant PD-L1 expression in Epstein-Barr Virus-infected gastric cancers. Oncotarget 7(22): 32925-32932.

Derks S, Nason KS, Liao X, Stachler MD, Liu KX, Liu JB, Sicinska E, Goldberg MS, Freeman GJ, Rodig SJ, Davison JM, Bass AJ (2015) Epithelial PD-L2 expression marks Barrett's esophagus and oesophageal adenocarcinoma. Cancer Immunol Res 3(10): 1123-1129.

Dieci MV, Criscitiello C, Goubar A, Viale G, Conte P, Guarneri V, Ficarra G, Mathieu MC, Delaloge S, Curigliano G, Andre F (2014) Prognostic value of tumour-infiltrating lymphocytes on residual disease after primary chemotherapy for triple-negative breast cancer: a retrospective multicenter study. Ann Oncol 25(3): 611-618.

Dong M, Wang H-Y, Zhao X-X, Chen J-N, Zhang Y-W, Huang Y, Xue L, H-G Li, Du H, Wu X-Y, Shao C-K (2016a) Expression and prognostic roles of PIK3CA, JAK2, PD-L1, and PD-L2 in Epstein-Barr virusassociated gastric carcinoma. Hum Pathol 53: 25-34.

Dong Y, Sun Q, Zhang X (2016b) PD-1 and its ligands are important immune checkpoints in cancer. Oncotarget 8(2): 2171-2186.

Edge SB, Compton CC (2010) The American Joint Committee on Cancer: the 7th edition of the AJCC cancer staging manual and the future of TNM. Ann Surg Oncol 17(6): 1471-1474.

Eto S, Yoshikawa K, Nishi M, Higashijima J, Tokunaga T, Nakao T, Kashihara H, Takasu C, Iwata T, Shimada M (2016) Programmed cell death protein 1 expression is an independent prognostic factor in gastric cancer after curative resection. Gastric Cancer 19(2): 466-471.

Fang W, Zhang J, Hong S, Zhan J, Chen N, Qin T, Tang Y, Zhang Y, Kang S, Zhou T, Wu X, Liang W, Hu Z, Ma Y, Zhao Y, Tian Y, Yang Y, Xue C, Yan Y, Hou X, Huang P, Huang Y, Zhao H, Zhang L (2014) EBV-driven LMP1 and IFN- $\gamma$ up-regulate PD-L1 in nasopharyngeal carcinoma: implications for oncotargeted therapy. Oncotarget 5(23): 12189-12202.

Festino L, Botti G, Lorigan P, Masucci GV, Hipp JD, Horak CE, Melero I, Ascierto PA (2016) Cancer treatment with anti-PD-1/PD-L1 agents: is PD-L1 expression a biomarker for patient selection? Drugs 76(9): 925-945.

Herbst RS, Soria JC, Kowanetz M, Fine GD, Hamid O, Gordon MS, Sosman JA, McDermott DF, Powderly JD, Gettinger SN, Kohrt HE, Horn L, Lawrence DP, Rost S, Leabman M, Xiao Y, Mokatrin A, Koeppen H, Hegde PS, Mellman I, Chen DS, Hodi FS (2014) Predictive correlates of response to the anti-PD-L1 antibody MPDL3280A in cancer patients. Nature 515(7528): 563-567.

Jin Z, Yoon HH (2016) The promise of PD-1 inhibitors in gastro-oesophageal cancers: microsatellite instability vs. PD-L1. J Gastrointest Oncol 7(5): 771-788.

Kang BW, Seo AN, Yoon S, Bae HI, Jeon SW, Kwon OK, Chung HY, Yu W, Kang H, Kim JG (2015) Prognostic value of tumour-infiltrating lymphocytes in Epstein-Barr virus-associated gastric cancer. Ann Oncol 27(3): 494-501.

Kang BW, Seo AN, Yoon S, Bae HI, Jeon SW, Kwon OK, Chung HY, Yu W, Kang H, Kim JG (2016) Prognostic value of tumour-infiltrating lymphocytes in Epstein-Barr virus-associated gastric cancer. Ann Oncol 27(3): 494-501.

Kawazoe A, Kuwata T, Kuboki Y, Shitara K, Nagatsuma AK, Aizawa M, Yoshino T, Doi T, Ohtsu A, Ochiai A (2016) Clinicopathological features of programmed death ligand 1 expression with tumour-infiltrating lymphocyte, mismatch repair, and Epstein-Barr virus status in a large cohort of gastric cancer patients. Gastric Cancer 20(3): 407-415.

Kim JW, Nam KH, Ahn S-H, Park DJ, Kim H-H, Kim SH, Chang H, Lee J-O, Kim YJ, Lee HS, Kim JH, Bang S-M, Lee JS, Lee K-W (2016) Prognostic implications of immunosuppressive protein expression in tumours as well as immune cell infiltration within the tumour microenvironment in gastric cancer. Gastric Cancer 19(1): 42-52.

Koh J, Ock C-Y, Kim JW, Nam SK, Kwak Y, Yun S, Ahn S-H, Park DJ, Kim H-H, Kim WH, Lee HS (2017) Clinicopathologic implications of immune classification by PD-L1 expression and CD8-positive tumourinfiltrating lymphocytes in stage II and III gastric cancer patients. Oncotarget 8(16): 26356-26367.

Latchman Y, Wood CR, Chernova T, Chaudhary D, Borde M, Chernova I, Iwai Y, Long AJ, Brown JA, Nunes R, Greenfield EA, Bourque K, 
Boussiotis VA, Carter LL, Carreno BM, Malenkovich N, Nishimura H, Okazaki T, Honjo T, Sharpe AH, Freeman GJ (2001) PD-L2 is a second ligand for PD-1 and inhibits T cell activation. Nat Immunol 2(3): 261-268.

Loke Pn, Allison JP (2003) PD-L1 and PD-L2 are differentially regulated by Th1 and Th2 cells. Proc Natl Acad Sci USA 100(9): 5336-5341.

Ma C, Patel K, Singhi AD, Ren B, Zhu B, Shaikh F, Sun W (2016) Programmed death-ligand 1 expression is common in gastric cancer associated with Epstein-Barr virus or microsatellite instability. Am J Surg Pathol 40(11): 1496-1506.

Manson G, Norwood J, Marabelle A, Kohrt H, Houot R (2016) Biomarkers associated with checkpoint inhibitors. Ann Oncol 27(7): 1199-1206.

McShane LM, Altman DG, Sauerbrei W, Taube SE, Gion M, Clark GM (2005) REporting recommendations for tumour MARKer prognostic studies (REMARK). Nat Clin Pract Oncol 2(8): 416-422.

Menon S, Shin S, Dy G (2016) Advances in cancer immunotherapy in solid tumours. Cancers 8(12): 106.

Ohigashi Y, Sho M, Yamada Y, Tsurui Y, Hamada K, Ikeda N, Mizuno T, Yoriki R, Kashizuka H, Yane K, Tsushima F, Otsuki N, Yagita H, Azuma M, Nakajima Y (2005) Clinical significance of programmed death-1 ligand-1 and programmed death-1 ligand-2 expression in human oesophageal cancer. Clin Cancer Res 11(8): 2947-2953.

Palucka AK, Coussens LM (2016) The basis of oncoimmunology. Cell 164(6): 1233-1247.

Pardoll DM (2012) The blockade of immune checkpoints in cancer immunotherapy. Nat Rev Cancer 12(4): 252-264.

Park J, Kwon M, Shin E-C (2016) Immune checkpoint inhibitors for cancer treatment. Arch Pharm Res 39(11): 1577-1587.

Saito R, Abe H, Kunita A, Yamashita H, Seto Y, Fukayama M (2016) Overexpression and gene amplification of PD-L1 in cancer cells and PD$\mathrm{L} 1+$ immune cells in Epstein-Barr virus-associated gastric cancer: the prognostic implications. Mod Pathol 30(3): 427-439.

Takano S, Saito H, Ikeguchi M (2016) An increased number of PD-1+ and Tim-3 + CD8 + T cells is involved in immune evasion in gastric cancer. Surg Today 46(11): 1341-1347.
Takaya S, Saito H, Ikeguchi M (2015) Upregulation of immune checkpoint molecules, PD-1 and LAG-3, on CD4 + and CD8 $+\mathrm{T}$ cells after gastric cancer surgery. Yonago Acta Med 58(1): 39-44.

Taube JM, Klein A, Brahmer JR, Xu H, Pan X, Kim JH, Chen L, Pardoll DM, Topalian SL, Anders RA (2014) Association of PD-1, PD-1 ligands, and other features of the tumour immune microenvironment with response to anti-PD-1 therapy. Clin Cancer Res 20(19): 5064-5074.

Teng MW, Ngiow SF, Ribas A, Smyth MJ (2015) Classifying cancers based on T-cell infiltration and PD-L1. Cancer Res 75(11): 2139-2145.

Wang S, Bajorath J, Flies DB, Dong H, Honjo T, Chen L (2003) Molecular modeling and functional mapping of B7-H1 and B7-DC uncouple costimulatory function from PD-1 interaction. J Exp Med 197(9): 1083-1091.

Wang X, Teng F, Kong L, Yu J (2016) PD-L1 expression in human cancers and its association with clinical outcomes. Oncotargets Ther 9: 5023-5039.

Wu C, Zhu Y, Jiang J, Zhao J, Zhang X-G, Xu N (2006) Immunohistochemical localization of programmed death-1 ligand-1 (PD-L1) in gastric carcinoma and its clinical significance. Acta Histochem 108(1): 19-24.

Zhang L, Qiu M, Jin Y, Ji J, Li B, Wang X, Yan S, Xu R, Yang D (2015) Programmed cell death ligand 1 (PD-L1) expression on gastric cancer and its relationship with clinicopathologic factors. Int J Clin Exp Pathol 8(9): 11084-11091.

Zhang M, Dong Y, Liu H, Wang Y, Zhao S, Xuan Q, Wang Y, Zhang Q (2016) The clinicopathological and prognostic significance of PD-L1 expression in gastric cancer: a meta-analysis of 10 studies with 1,901 patients. Sci Rep 6: 37933.

Zhang Y, Chung Y, Bishop C, Daugherty B, Chute H, Holst P, Kurahara C, Lott F, Sun N, Welcher AA, Dong C (2006) Regulation of T cell activation and tolerance by PDL2. Proc Natl Acad Sci USA 103(31): 11695-11700.

This work is published under the standard license to publish agreement. After 12 months the work will become freely available and the license terms will switch to a Creative Commons AttributionNonCommercial-Share Alike 4.0 Unported License.

Supplementary Information accompanies this paper on British Journal of Cancer website (http://www.nature.com/bjc) 